\title{
醂
}

Diego Quer Ramón*

Enrique Claver Cortés*

Laura Rienda García*

\section{LAS MULTINACIONALES CHINAS EN ESPAÑA Situación actual y retos de futuro}

Las multinacionales chinas se están convirtiendo en actores principales del nuevo escenario global, y han realizado inversiones significativas en otros países durante los últimos años. Aunque España todavía no es uno de sus destinos principales, se observa una tendencia creciente de los flujos inversores. Este trabajo estudia las características de las inversiones chinas en España con datos a nivel de empresa, analizando el número de inversiones, los principales sectores de destino, la propiedad estatal o privada de la empresa china inversora, la modalidad de inversión utilizada y la región de localización. Asimismo, ofrece una serie de reflexiones sobre la importancia de la inversión china en España y sus perspectivas de cara al futuro.

Palabras clave: inversión china en el exterior, España.

Clasificación JEL: F23, M16.

\section{Introducción}

Durante las tres últimas décadas, empresas de todo el mundo han considerado a China como un destino preferente para la inversión directa en el exterior (IDE). Al principio, sólo para explotar ventajas de coste y, más recientemente, cada vez más para aprovechar las oportunidades que ofrece su enorme mercado. Sin embargo, China ya no sólo es uno de los principales receptores de IDE a nivel mundial, sino que se está consolidando también como uno de los principales países emisores.

En los últimos años, las empresas chinas han efectuado inversiones significativas en otros países. Algunas han supuesto la adquisición de emblemáticas marcas occidentales, como las norteamericanas IBM y Motorola (por parte de Lenovo) o la sueca Volvo (ahora en manos de Geely

* Departamento de Organización de Empresas, Universidad de Alicante.

Versión de mayo de 2015.
Automobile). Sin embargo, las operaciones que han implicado un mayor volumen de inversión se han centrado en el sector energético, destacando la adquisición de la canadiense Nexen por parte de China Offshore Oil Corporation (CNOOC) o la compra de la también canadiense Addax Petroleum y de un 40 por 100 de la filial brasileña de Repsol por parte de Sinopec. Una de las consecuencias más inmediatas de este crecimiento de la IDE china es el creciente papel protagonista que las multinacionales chinas están adquiriendo en el escenario global. Como botón de muestra, cabe destacar que ya hay 95 empresas chinas entre las 500 más grandes del mundo por volumen de facturación (sólo por detrás de EEUU que cuenta con 128 empresas) y tres de ellas (Sinopec, CNPC y State Grid) están entre las diez primeras (Fortune, 2014).

Los factores motivadores de la IDE efectuada por empresas chinas presentan una serie de rasgos distintivos (Deng, 2004; Hong y Sun, 2006; $\triangleright$ 
Quer, Claver y Rienda, 2007, 2013; Wong y Chan, 2003; Wu y Sia, 2002). La búsqueda de eficiencia mediante la localización de determinadas actividades en países de menor coste no ha sido uno de los motivos habituales (debido a las ventajas de coste que tradicionalmente han disfrutado en su país de origen). Por el contrario, la búsqueda de recursos (petróleo, gas, metal, etcétera) ha sido uno de los objetivos tradicionales con la finalidad de satisfacer las necesidades derivadas del desarrollo económico de China, esencialmente basado en actividades industriales. La búsqueda de mercados también ha sido otro de sus objetivos, estableciendo filiales comerciales para fomentar las exportaciones desde China (otro de los ejes fundamentales de su desarrollo económico) o incluso filiales productivas (para soslayar restricciones a la exportación en algunos destinos).

No obstante, en los últimos años la búsqueda de activos estratégicos está cobrando una importancia creciente como factor motivador de la IDE china. Las multinacionales chinas han iniciado su proceso de internacionalización mucho más tarde que otras procedentes de países desarrollados, por lo que necesitan acceder a recursos intangibles como marca, tecnología o know-how para situarse al mismo nivel de esos competidores establecidos (Luo y Tung, 2007; Meyer, 2015; Yang, Yang, Chen y Allen, 2014). Una forma rápida de acceder a esos activos estratégicos es mediante la adquisición de empresas que ya disponen de ellos y que habitualmente están localizadas en países desarrollados (Child y Rodrigues, 2005). Las adquisiciones mencionadas de IBM, Motorola o Volvo son ejemplos significativos de esta estrategia.

España todavía no es un destino primordial para la IDE china. Sin embargo, en el último lustro las inversiones chinas en España están creciendo de manera exponencial ya que importantes multinacionales chinas como Huawei, Lenovo, ZTE o Dalian Wanda Group han empezado a efectuar inversiones relevantes en España, esencialmente buscando mercados o activos estratégicos.
Hasta el momento, existe un escaso número de trabajos sobre las inversiones chinas en España. Carril y Milgram (2014) han efectuado un análisis de los factores determinantes de la inversión a partir de una encuesta a 30 empresas localizadas en España con participación china. Sus resultados desvelan que los principales motivos de la IDE china en España son la búsqueda de activos estratégicos (en particular, marcas reconocidas) y de mercados (dado que desarrollan en España principalmente actividades comerciales o de servicios).

Cabe destacar también el estudio de ESADE (2014) que, a partir de una encuesta a 22 empresas chinas establecidas en España, confirma también que los motivos principales para invertir en España son: la búsqueda de mercados (en particular, abrir nuevos mercados mediante presencia física) y de activos estratégicos (principalmente, acceso a canales de distribución, ser identificadas como empresas locales y acceder a personal cualificado). Asimismo, las empresas encuestadas consideraron los recursos humanos (calidad, disponibilidad y actitud hacia China) como la variable mejor valorada del clima de negocios español, mientras que reconocieron que la falta de reconocimiento de marca es su principal desafío en España.

Por último, cabe mencionar otros estudios de naturaleza descriptiva realizados por Goy y Navarro (2008), Hernández y Álvarez (2009), Sáez (2010) y Santacana y Wang (2008). Dichos trabajos analizaron la evolución de la inversión china en España con datos agregados hasta 2006-2008. De estos trabajos, podemos extraer algunas conclusiones: la evolución anual de los flujos de inversión china en España fue desigual durante ese periodo, estando condicionada por inversiones puntuales con un impacto desproporcionado; existía un sesgo hacia la localización en Cataluña; y España, por su situación geográfica y lazos históricos, económicos y culturales, puede servir como plataforma de acceso para hacer negocios en Latinoamérica, Norte de África y el área mediterránea.

El objetivo de nuestro trabajo es realizar una aproximación a la realidad actual de las inversiones chinas en España y sus perspectivas de futuro. Para ello, partiendo de una base de datos propia $D$ 

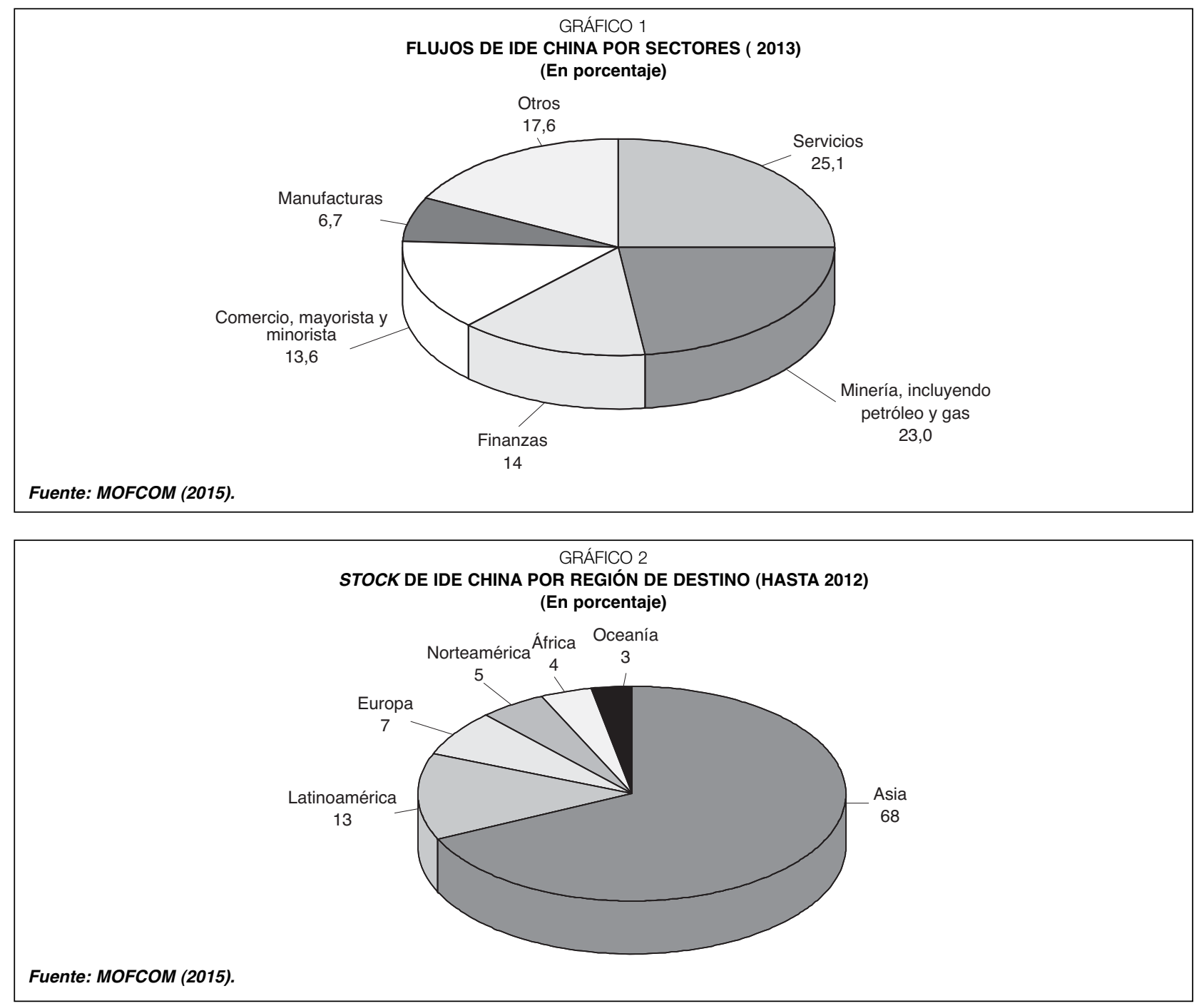

elaborada a partir de diversas fuentes de información, vamos a ofrecer una descripción de la IDE china en España con datos a nivel de empresa. Entre otras cuestiones, vamos a analizar cuáles son las multinacionales chinas con presencia en España, su tamaño, sus sectores de actividad, su carácter estatal o privado, las regiones españolas donde se han localizado o la modalidad que han empleado para implantarse en España. Antes de ello, vamos a ofrecer una panorámica general agregada de la IDE china.

\section{La IDE china: visión general e importancia de España como destino}

El crecimiento de los flujos de IDE emitidos por China ha sido espectacular en los últimos años, multiplicándose por cuatro sólo entre 2007 y 2014. Según datos de la UNCTAD (2015), la IDE china alcanzó los 116.000 millones de dólares en 2014, lo que convierte a China continental en el tercer inversor del mundo, sólo superado por Hong Kong (China), que ocupa el segundo lugar, y EEUU, que encabeza el ranking.

Desde un punto de vista agregado, la mayor parte de la IDE china se centra en el sector servicios y en el de la minería (incluyendo petróleo y gas), tal y como recoge el Gráfico 1. Respecto a la localización, existe un claro sesgo hacia las inversiones en Asia, que aglutinan casi un 70 por 100 del stock acumulado (Gráfico 2). No obstante, las estadísticas oficiales hacen difícil conocer exactamente el destino final de muchas de las inversiones chinas, ya $\triangleright$ 

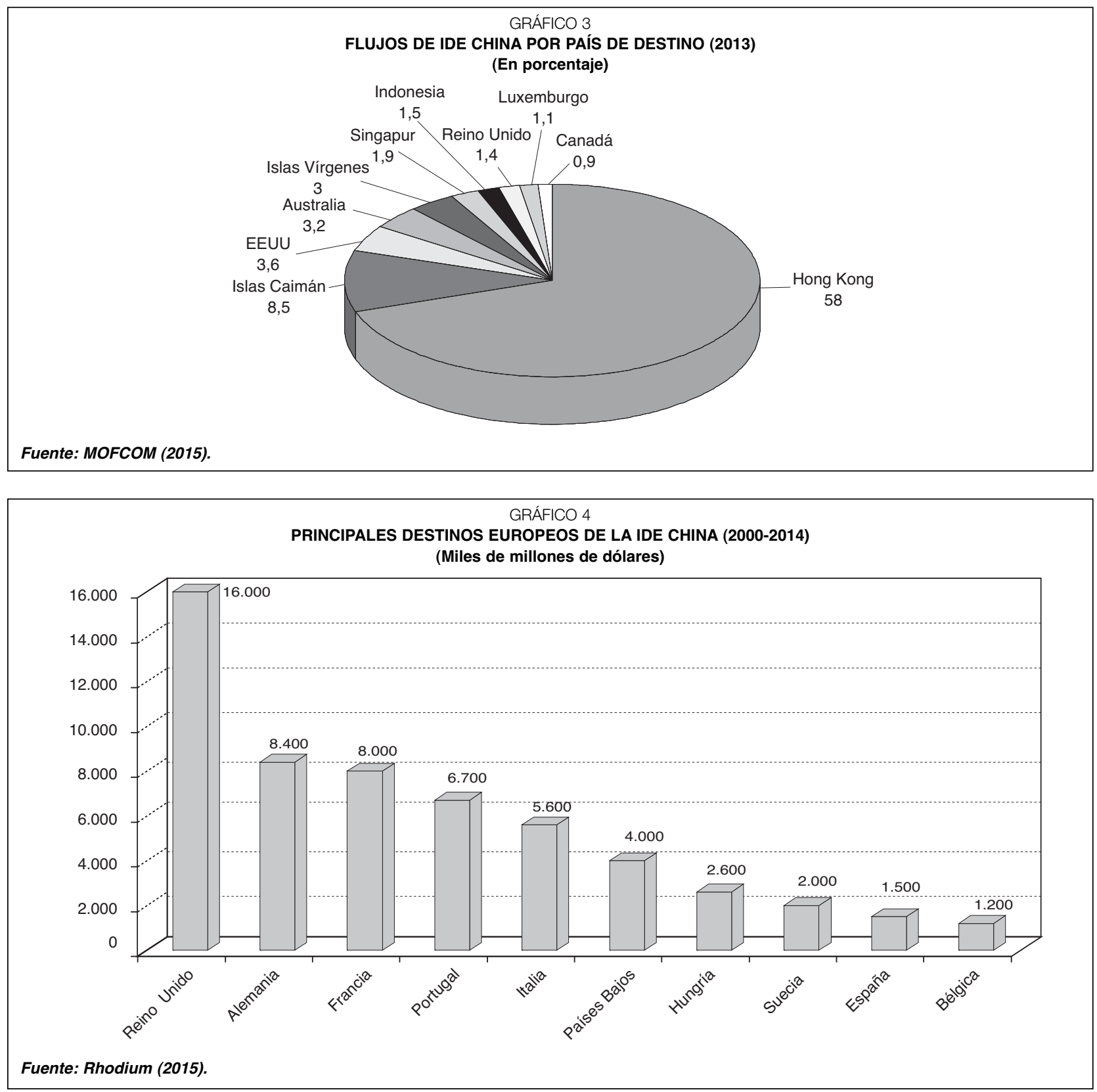

que Hong Kong se sitúa como el primer destino, cuando se trata de una Región Administrativa Especial de la República Popular China y algunas de estas inversiones pueden incluso volver a la propia China continental, aprovechando las posibles ventajas derivadas de invertir desde Hong Kong (es lo que se denomina round-tripping). Además, paraísos fiscales como Islas Caimán e Islas Vírgenes Británicas también aparecen como destinos relevantes (Gráfico 3).

Por lo que respecta a Europa, según un informe elaborado por Rhodium Group y Baker \& McKenzie
(Rhodium, 2015), la IDE china ha ido creciendo significativamente desde el inicio del siglo XXI, especialmente desde 2010, hasta alcanzar los 18.000 millones de dólares en 2014 , el doble que un año antes. De acuerdo a dicho informe, Reino Unido es, con diferencia, el país de la Unión Europea que ha atraído el mayor volumen de inversiones chinas desde el año 2000, seguido de Alemania y Francia (Gráfico 4).

Según las estadísticas oficiales del Gobierno de España (Datalnvex, 2015), la inversión china en España empieza a despegar sólo a partir del año $D$ 


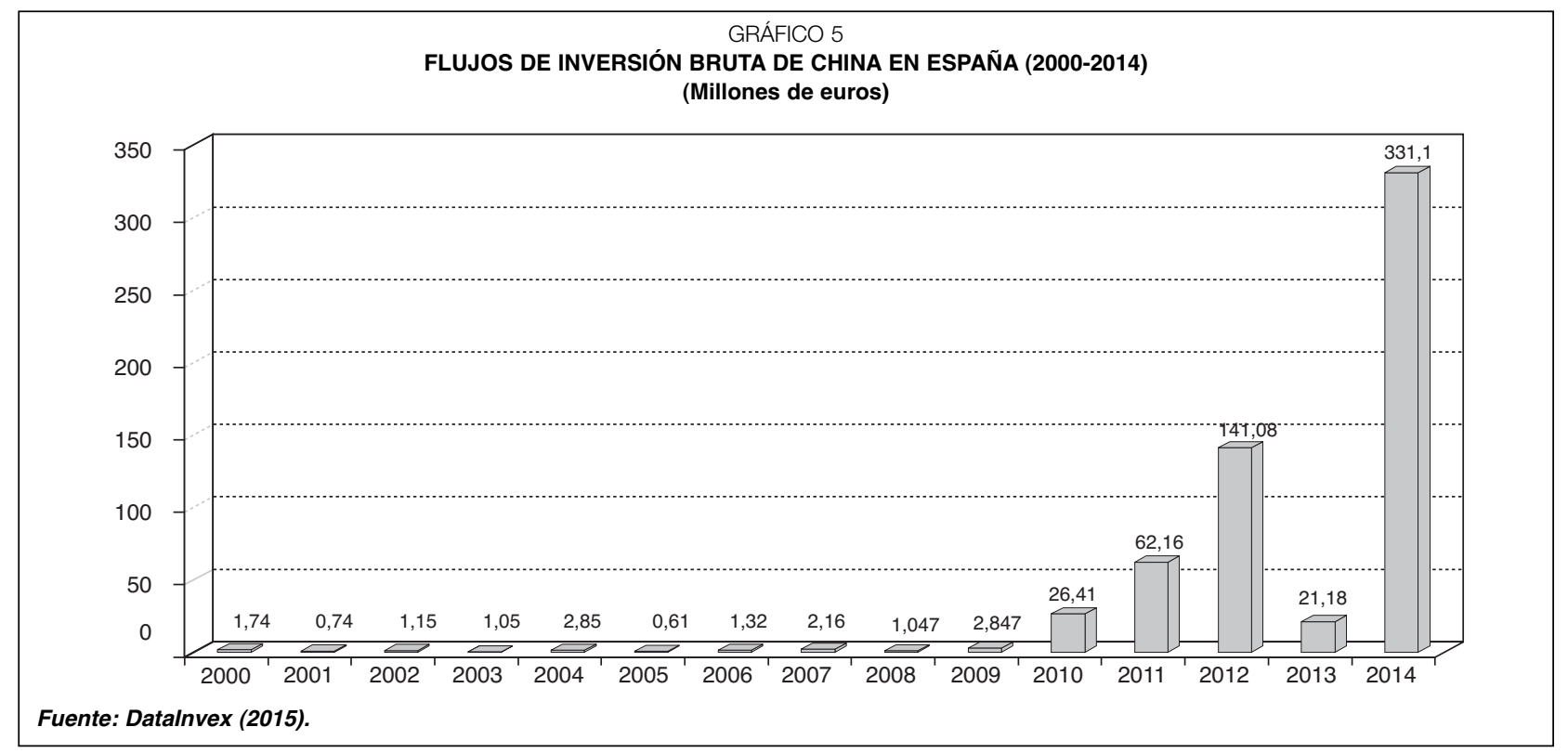

2010, alcanzando su máximo histórico en 2014, con 331 millones euros, representando un 1,88 por 100 del total de IDE recibida por España ese año (Gráfico 5). No obstante, los datos reflejados por Datalnvex deben ser tomados con cautela, ya que algunos flujos anuales pueden venir condicionados por adquisiciones significativas puntuales y las inversiones de algunas empresas chinas pueden no estar recogidas (ESADE, 2014). Cabe destacar que los datos recogidos en el Gráfico 5 se refieren a inversiones procedentes de China continental, sin considerar algunas que hayan podido ser canalizadas desde Hong Kong.

Pese a que estas cifras denotan que, a nivel agregado, la inversión china en España todavía no es muy elevada, lo cierto es que en los últimos años estamos asistiendo al desembarco de importantes multinacionales chinas. Algunos ejemplos destacados son la empresa de equipamiento de telecomunicaciones Huawei (sobre todo, gracias a su alianza estratégica con Telefónica), el grupo turístico HNA (que compró un 29,5 por 100 de la cadena hotelera $\mathrm{NH}$ ), la empresa de alimentación Shuanghui, actualmente rebautizada como WH Group (que posee un 37 por 100 de Campofrío), el grupo inversor Fosun (que controla un 20 por 100 de Osborne), los bancos ICBC y China Construcción Bank (que tienen sucursales en España), el conglomerado Dalian Wanda Group (que adquirió el emblemático Edificio España en Madrid y un 20 por 100 del capital del Atlético de Madrid), la empresa de equipos de telecomunicaciones ZTE (que estableció en España su sede para todo el sur de Europa) o las conocidas marcas chinas Lenovo y Haier (que cuentan con una creciente presencia comercial en España, la primera esencialmente en el sector de equipos informáticos y la segunda en el de electrodomésticos).

\section{Multinacionales chinas en España: análisis con datos a nivel de empresa}

A continuación ofrecemos un análisis con datos a nivel de empresa sobre las multinacionales chinas que han invertido en España. Para ello, hemos elaborado una base de datos propia a partir de distintas fuentes de información: listado de empresas chinas implantadas en España elaborado por el Observatorio ESADE China Europe Club (ESADE, 2014), empresas con sede en España que cuentan con accionistas de China o Hong Kong según la base de datos SABI (Sistema de Análisis de Balances lbéricos), y otros listados de empresas chinas en España proporcionados por la Cámara $\triangleright$ 


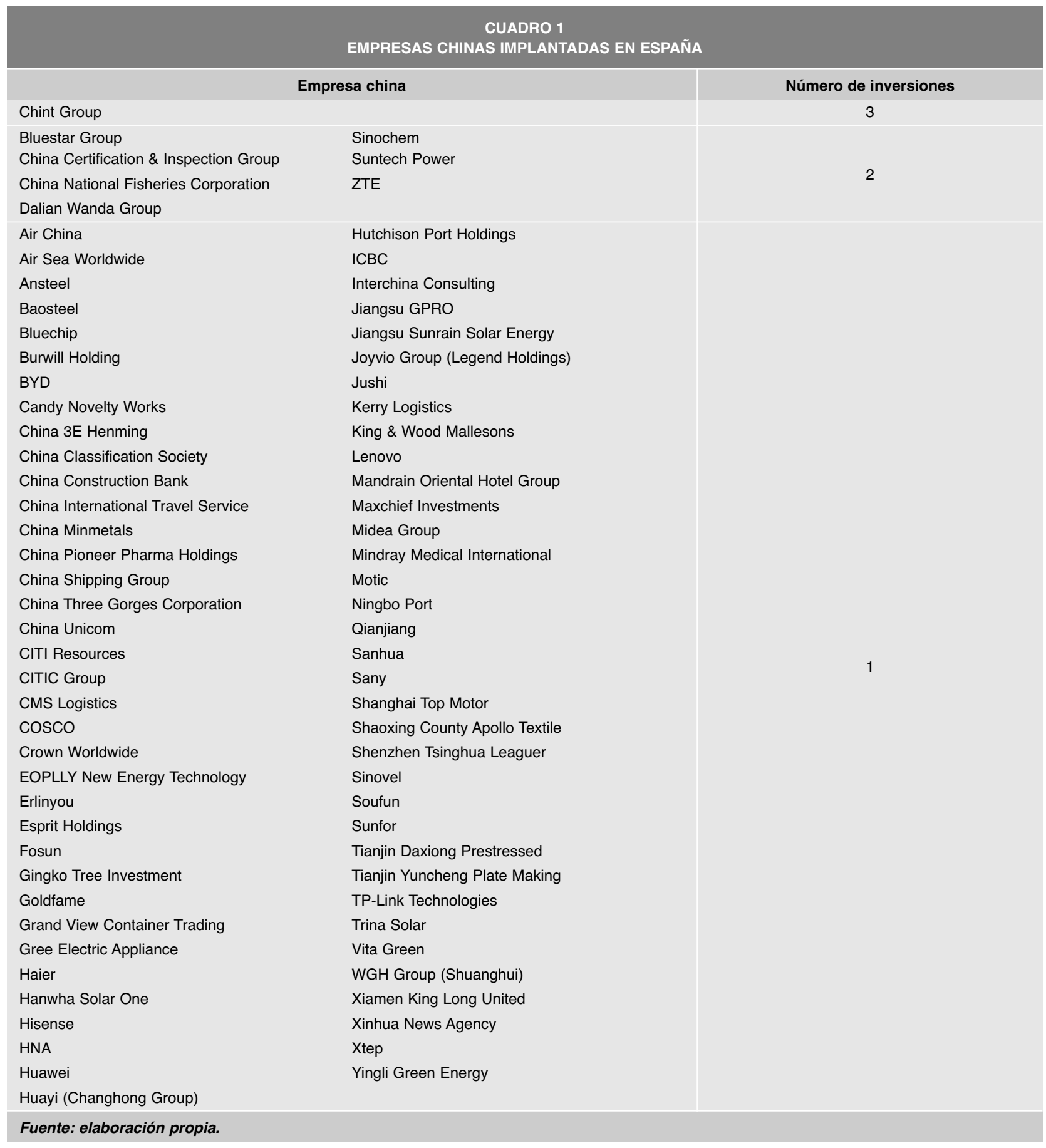

de Comercio e Inversiones de China en España y por la Oficina del Consejero Económico-Comercial de la Embajada de la República Popular China en España.

Tras cruzar la información procedente de todas esas fuentes, cotejamos y depuramos los datos mediante la consulta de las páginas web corporativas de las empresas y de noticias aparecidas en medios de comunicación tanto chinos como españoles hasta mayo de 2015. Finalmente, identificamos $88 \mathrm{em}-$ presas establecidas en España que cuentan con participación china (Cuadro 1).

Como se observa en el Cuadro 1, la mayoría de empresas chinas ha realizado una única inversión en España. El ranking lo encabeza Chint Group, empresa de equipamiento eléctrico y energía solar $D$ 


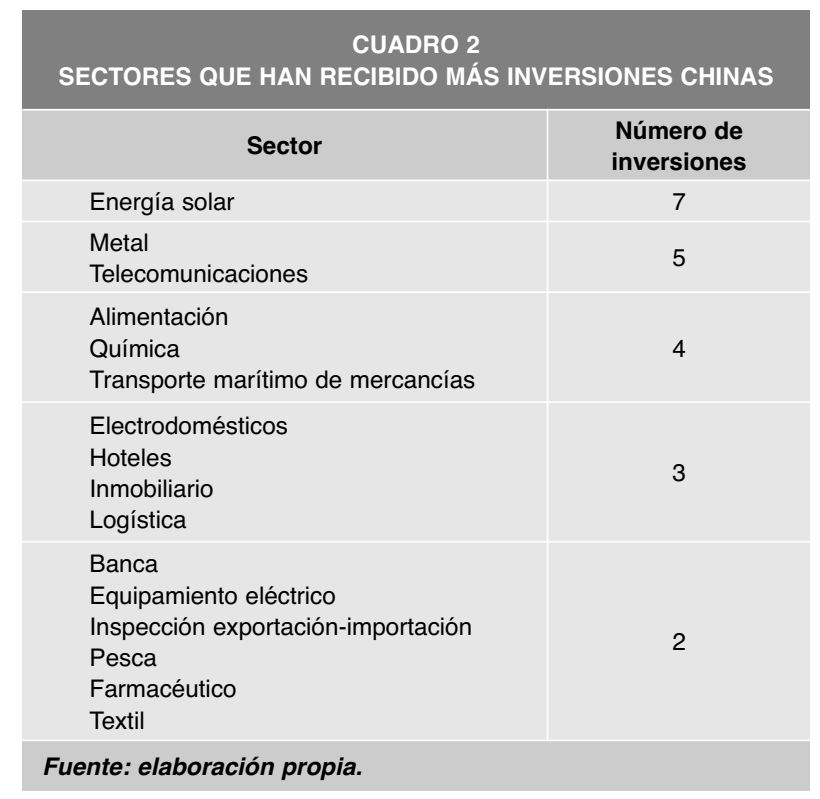

(que cuenta con 3 inversiones). A continuación encontramos a Bluestar Group, China Certification \& Inspection Group, China National Fisheries Corporation, Dalian Wanda Group, Sinochem, Suntech Power y ZTE (que han realizado 2 inversiones cada una).

Respecto a la estructura de propiedad de la empresa inversora, destaca un predominio de las inversiones realizadas por empresas chinas de capital privado (57 inversiones), que representan un 64,8 por 100 , mientras que las 31 inversiones restantes $(35,2$ por 100$)$ han sido efectuadas por empresas chinas de propiedad estatal. Por otra parte, la mayoría de las multinacionales chinas identificadas en nuestra base de datos tienen su sede central en las regiones más desarrolladas y con mayor grado de apertura exterior de China. Concretamente, la mayoría proceden de Beijing (sede central de 28 empresas inversoras), seguida de Hong Kong (15), Guangdong (10), Shanghai (9), Jiangsu (7) y Zhejiang (5). En conjunto, estas 6 regiones suponen el 84,1 por 100 de nuestras observaciones.

En cuanto a la distribución sectorial, predominan claramente las inversiones de naturaleza comercial o de servicios (73 inversiones, un 83 por $100)$ sobre las manufactureras (15 inversiones, un 17 por 100). El sector que aglutina más inversiones ha sido el de energía solar (7), seguido de metal y telecomunicaciones (5 cada uno) y alimentación, química y transporte marítimo de mercancías (4 cada uno). El Cuadro 2 recoge la distribución sectorial de las inversiones, considerando sólo aquellos sectores que han recibido más de una inversión.

Como se observa en el Gráfico 6, la inversión más antigua recogida en nuestra base de datos se remonta a 1993, aunque la mayor parte se han efectuado en los últimos años. Concretamente, hemos identificado 64 inversiones desde 2007, lo que representa un 72,7 por 100 del total, y 45 se han realizado desde 2010, lo que supone más de la mitad del total (un 51,1 por 100).

Desde el punto de vista geográfico, se observa un sesgo muy importante, ya que un 81,8 por 100 de las inversiones se ha concentrado en sólo dos provincias: Madrid (que ha recibido 39 inversiones) y Barcelona (33 inversiones). El Cuadro 3 recoge la distribución de las inversiones chinas por provincias.

Finalmente, en cuanto al tamaño empresarial, como se observa en el Gráfico 7, predominan las empresas de menor dimensión. Concretamente, un 55,3 por 100 son empresas que no sobrepasan los 10 empleados, sólo un 21,1 por 100 tiene más de 50 y únicamente 4 empresas están por encima de los 500 .

\begin{tabular}{|c|c|}
\hline \multicolumn{2}{|c|}{$\begin{array}{c}\text { CUADRO } 3 \\
\text { DISTRIBUCIÓN DE LA INVERSIÓN CHINA EN ESPAÑA } \\
\text { POR PROVINCIAS }\end{array}$} \\
\hline Provincia & $\begin{array}{l}\text { Número de } \\
\text { inversiones }\end{array}$ \\
\hline Madrid & 39 \\
\hline Barcelona & 33 \\
\hline $\begin{array}{l}\text { Burgos } \\
\text { A Coruña } \\
\text { Las Palmas }\end{array}$ & 2 \\
\hline $\begin{array}{l}\text { Albacete } \\
\text { Alicante } \\
\text { Asturias } \\
\text { Cádiz } \\
\text { Guadalajara } \\
\text { Mallorca } \\
\text { Pontevedra } \\
\text { Tarragona } \\
\text { Valencia } \\
\text { Vizcaya }\end{array}$ & 1 \\
\hline
\end{tabular}



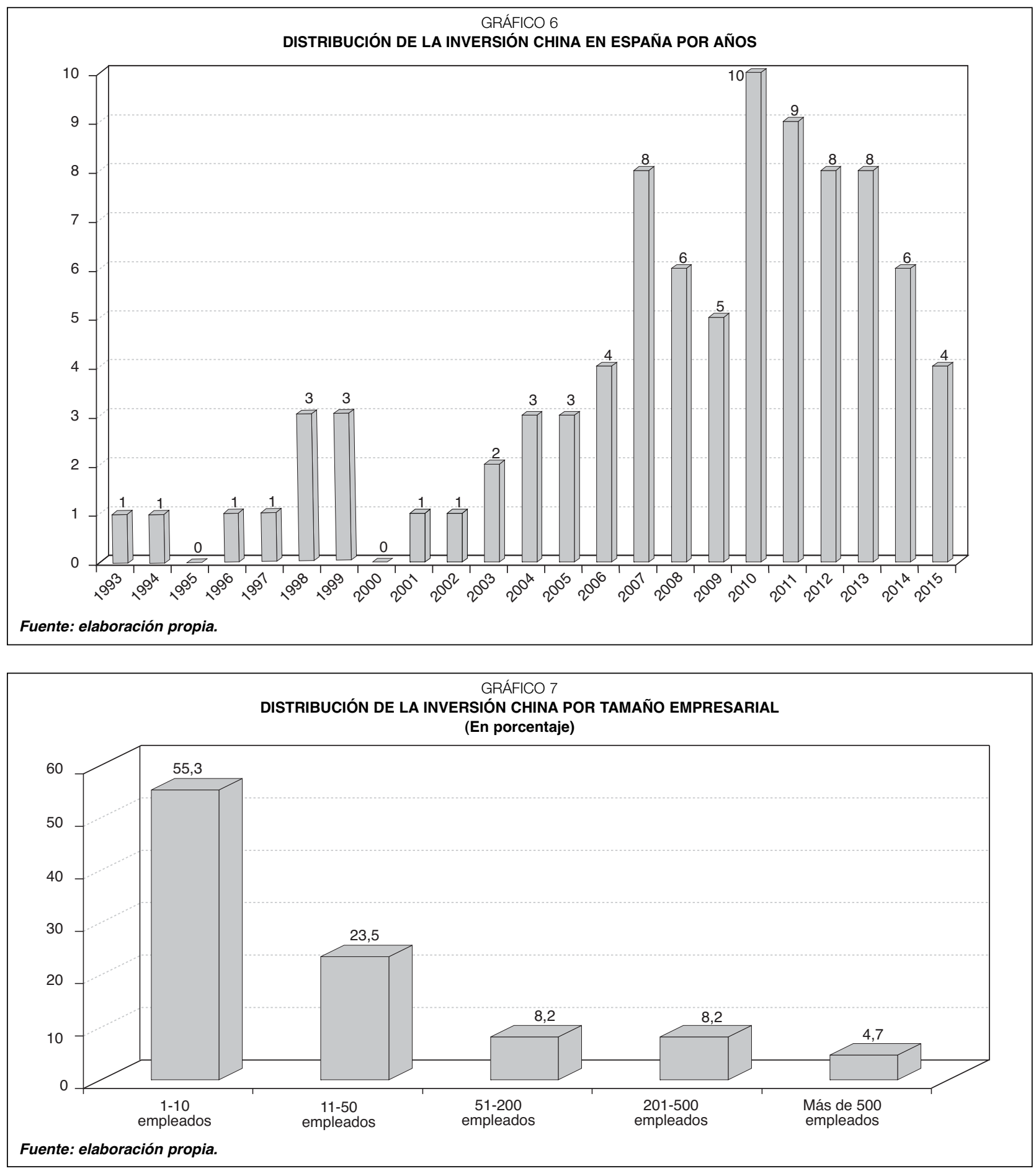

\section{Conclusiones}

Las multinacionales chinas están protagonizando un acelerado proceso de internacionalización en estas primeras décadas del nuevo siglo, favorecidas por una serie de factores. En primer lugar, por la disponibilidad de recursos financieros para efectuar IDE (debido sobre todo a la enorme cantidad de divisas acumuladas por China tras varias décadas de desarrollo económico orientado a la exportación). En segundo lugar, gracias al conocimiento adquirido de las empresas extranjeras establecidas en China, muchas de las cuales establecieron joint ventures allí con $\triangleright$ 
socios locales que hoy día se han convertido en esas grandes multinacionales chinas. Finalmente, por el apoyo del propio Gobierno chino, una de cuyas líneas de actuación se centra en fomentar la competitividad internacional de las empresas chinas, por lo que ha ido reduciendo progresivamente los obstáculos para que efectúen IDE.

Por otra parte, existe un creciente interés de las instituciones y empresas españolas por atraer inversiones procedentes de China, ya que pueden tener consecuencias positivas en términos de empleo y generación de riqueza. Un ejemplo de ello lo encontramos en Cubigel, empresa catalana fabricante de compresores para refrigeración, que en 2012 se encontraba en dificultades cuando fue adquirida por Huayi (perteneciente al grupo Changhong, uno de los principales fabricantes de TV de China). Dicha inversión contribuyó a mantener 406 puestos de trabajo.

Los lazos comerciales bilaterales entre España y China se han intensificado en los últimos años. El tren de mercancías entre Yiwu (Zhejiang) y Madrid, considerado como el más largo del mundo, es un buen ejemplo de ello. Aunque completó su primera ruta en diciembre de 2014 y todavía está en fase experimental, se puede considerar parte integrante del ambicioso proyecto del Gobierno chino anunciado en 2013 de revitalizar la antigua Ruta de la Seda. Esta iniciativa pretende desarrollar una gran red de transporte y comunicaciones que comunique Asia con Europa, mediante dos vías: una terrestre (conocida como el "Cinturón Económico de la Ruta de la Seda») y otra marítima (bautizada como la «Ruta Marítima de la Seda del siglo XX|»). Por otra parte, en abril de 2015, España se convirtió en uno de los primeros países europeos en ser admitido como miembro fundador del Banco Asiático de Inversión en Infraestructuras auspiciado por China. Este banco de desarrollo multilateral pretende financiar proyectos de infraestructuras en Asia, aunque el apoyo explícito de España puede allanar el camino también para futuras inversiones chinas en España.

Lógicamente, todo este proceso de expansión internacional de las multinacionales chinas no está exento de retos. Muchas son empresas de reciente internacionalización, por lo que es posible que les falte experiencia en adquisiciones internacionales, sobre todo para una adecuada integración de las empresas adquiridas. En el caso de Europa, dicho obstáculo se acrecienta por las diferencias culturales que intensifican la «desventaja del extranjero" para las empresas chinas (Brennan, 2015). Entre otras cuestiones, deben adaptar su estilo de organización y de dirección, tradicionalmente muy jerárquico, a la cultura europea, donde existe una preferencia por una mayor autonomía.

Asimismo, algunas de estas multinacionales chinas son empresas estatales, por lo que sus inversiones en países desarrollados despiertan en ocasiones recelos por parte de los Gobiernos anfitriones. Pero sin duda, uno de sus mayores obstáculos es la falta de imagen de marca con reconocimiento internacional. En este sentido, resulta interesante el caso de Huawei en España. Aparte de su apuesta por estar presente en el sector del fútbol, lo que le reporta una gran visibilidad, destaca su alianza estratégica con operadores como Telefónica, a los que proporciona entre otros productos, teléfonos móviles, que son usados por los clientes españoles. Ello puede ayudar a generar una imagen favorable de los productos chinos, rompiendo con los estereotipos de producto de bajo precio y escasa calidad, y originar un posible efecto arrastre para otras empresas chinas que quieran instalarse en España.

\section{Bibliografía}

[1] BRENNAN, L. (2015). «The challenges for Chinese FDI in Europe». Columbia FDI Perspectives. Perspectives on topical foreign direct investment issues by the Vale Columbia Center on Sustainable International Investment, $\mathrm{n}^{\circ}$ 142, 2 de marzo 2015.

[2] CARRIL, F. y MILGRAM, J. (2014). "Chinese outward FDI in Spain: An analysis of the determinants at the firm level». XI INTECO Workshop on Economic Integration, Valencia, noviembre. $D$ 
[3] CHILD, J. y RODRIGUES, S.B. (2005). «The internationalization of Chinese firms: A case for theoretical extension?». Management and Organization Review, vol. 1, n 3, pp. 381-410.

[4] DATAINVEX (2015). Inversión extranjera en España. Datalnvex Estadísticas de Inversión Extranjera en España, [en línea]. Madrid: Secretaría de Estado de Comercio. Ministerio de Economía y Competitividad. Gobierno de España. Disponible en:

datainvex.comercio.es [Recuperado en mayo de 2015].

[5] DENG, P. (2004). "Outward investment by Chinese MNCs: Motivations and implications». Business Horizons, vol. 47, ${ }^{\circ} 3$, pp. 8-16.

[6] ESADE (2014): Inversión china en Europa 2014. Barcelona: ESADE China Europe Club.

[7] FORTUNE (2014). Global 500 2014, [en línea]. New York: Time Inc. Disponible en:

fortune.com/global500 [Recuperado en mayo de 2015].

[8] GOY, A.M. y NAVARRO, A. (2008). ¿Cómo fomentar la inversión asiática en España? Madrid: Observatorio de Política Exterior Española. Fundación Alternativas. Documento de Trabajo 18/2008.

[9] HERNÁNDEZ, A. y ÁlVAREZ, O. (2009). «China, nuevo actor de la inversión extranjera global». Boletín Económico de ICE, n 2972, pp. 165-175.

[10] HONG, E. y SUN, L. (2006). «Dynamics of internationalization and outward investment: Chinese corporations' strategies». The China Quarterly, $n^{\circ} 187$, pp. 610-634.

[11] LUO, Y. y TUNG, R.L. (2007). «International expansion of emerging-market enterprises: $A$ springboard perspective». Journal of International Business Studies, vol. 38, n 4, pp. 481-498.

[12] MEYER, K.E. (2015): «What is "strategic asset seeking FDI"?». The Multinational Business Review, vol. 23, nº 1, pp. 57-66.
[13] MOFCOM (2015). Statistics, [en línea]. Beijing: Ministry of Commerce (MOFCOM) People's Republic of China. Disponible en: english.mofcom. gov.cn [Recuperado en mayo de 2015].

[14] QUER, D., CLAVER, E. y RIENDA, L. (2007). "Multinacionales chinas. El nuevo reto del escenario global”. Boletín Económico de ICE, $n^{\circ}$ 2925, pp. 11-24.

[15] QUER, D., CLAVER, E. y RIENDA, L. (2013). «La elección entre filial propia y joint venture: el caso de las multinacionales chinas». Información Comercial Española. Revista de Economía, $\mathrm{n}^{\circ}$ 870, pp. 131-146.

[16] RHODIUM (2015). Chinese investment into Europe. Reaching new heights, [en línea]. Rhodium Group/Baker \& McKenzie. Disponible en: bakermckenzie.com [Recuperado en mayo de 2015].

[17] SÁEZ, L. (2010). Chinese outward direct investments in Spain. London: Chatam House. International Economics Programme Paper 2010/06.

[18] SANTACANA, R. y WANG, Y.J. (2008). «Privatización e internacionalización: las empresas chinas en España». IX Congreso Internacional de la Asociación Española de Historia Económica, Murcia, septiembre.

[19] UNCTAD (2015). "Global investment trend monitor». United Nations Conference on Trade and Development, $\mathrm{n}^{\circ} 19$, mayo de 2015.

[20] WONG, J. y CHAN, S. (2003). "China's outward direct investment: Expanding worldwide». China: An International Journal, volumen $1, \mathrm{n}^{\circ} 2$, pp. 273-301.

[21] WU, F. y SIA, Y.H. (2002). "China's rising investment in Southeast Asia: Trends and outlook». Journal of Asian Business, vol. 18, $n^{\circ}$ 2, pp. 41-61.

[22] YANG, Y., YANG, X., CHEN, R. y ALLEN, J.P. (2014). «What drives emerging-economy outbound FDI decisions to obtain strategic assets?». Asian Business \& Management, vol. 13, $\mathrm{n}^{\circ} 5$, pp. 379-410. 